\title{
Nonlinear MHD dynamo operating at equipartition
}

\author{
V. Archontis ${ }^{1}$, S. B. F. Dorch ${ }^{2,3}$, and A. Nordlund ${ }^{2}$ \\ ${ }^{1}$ School of Mathematics and Statistics, University of St. Andrews, North Haugh, St. Andrews, Fife KY16 9SS, UK \\ e-mail: vasilis@ll.iac.es \\ 2 The Niels Bohr Institute, Juliane Maries Vej 30, 2100 Copenhagen Ø, Denmark \\ 3 Copenhagen University Library, The Royal Library, Nørre allé 49, 2200 Copenhagen N, Denmark
}

Received 24 February 2006 / Accepted 2 July 2007

\begin{abstract}
Context. We present results from non linear MHD dynamo experiments with a three-dimensional steady and smooth flow that drives fast dynamo action in the kinematic regime. In the saturation regime, the system yields strong magnetic fields, which undergo transitions between an energy-equipartition and a turbulent state. The generation and evolution of such strong magnetic fields is relevant for the understanding of dynamo action that occurs in stars and other astrophysical objects.

Aims. We study the mode of operation of this dynamo, in the linear and non-linear saturation regimes. We also consider the effect of varying the magnetic and fluid Reymolds number on the non-linear behaviour of the system.

Methods. We perform three-dimensional non-linear MHD simulations and visualization using a high resolution numerical scheme. Results. We find that this dynamo has a high growth rate in the linear regime, and that it can saturate at a level significantly higher than intermittent turbulent dynamos, namely at energy equipartition, for high values of the magnetic and fluid Reynolds numbers. The equipartition solution however does not remain time-independent during the simulation but exhibits a much more intricate behaviour than previously thought. There are periods in time where the solution is smooth and close to energy-equipartition and others where it becomes turbulent. Similarities and differences in the way the magnetic field is amplified and sustained for experiments with varying Reynolds numbers are discussed.

Conclusions. Strong magnetic fields, in near equipartition, can be generated also by a non-turbulent dynamo. A striking result is that the saturation state of this dynamo reveals interesting transitions between turbulent and laminar states.
\end{abstract}

Key words. magnetic fields - magnetohydrodynamics (MHD) - plasmas - turbulence

\section{Introduction}

Magnetic fields are ubiquitous in astrophysical bodies throughout the cosmos. They vary in scale and strength from strong magnetic fields in e.g. solar active regions to relatively weak magnetic fields in planets and galaxies. These fields are controlled by the motions of plasmas through a transfer of kinetic energy to magnetic energy: the resulting amplification and regeneration of the magnetic field is called dynamo action (Cowling 1934; Moffatt 1978; Parker 1979). The study of dynamo action in the limit of infinitely high magnetic Reynolds number $(\mathrm{Rm}=U \ell / \eta$, $U$ and $\ell$ are characteristic velocity and length scales and $\eta$ is the magnetic diffusivity) is the subject of fast dynamo theory and is relevant to most astrophysical systems, where the diffusion timescales are typically much larger than the advection timescales.

Considering the importance of the magnetic forces relative to the motion of the fluid, one divides dynamo action into two regimes: the linear kinematic regime in which the flow amplifies the magnetic field exponentially by e.g. stretching the magnetic field lines, and the non-linear regime where the magnetic field becomes strong enough to modify the initial flow topology through the Lorentz force, and consequently halts the exponential growth. Thorough reviews of dynamo theory may be found e.g. in Childress \& Gilbert (1995), and in Brandenburg \& Subramanian (2005).

Kinematic dynamos have been studied extensively in the past (recent examples are Dorch 2000; Schekochihin et al. 2002; Archontis et al. 2003a; Mininni et al. 2005a); these studies have improved our understanding of the exponential amplification of weak magnetic fields by prescribed flows. However, knowledge of the exact mechanisms behind the maintenance of magnetic fields against resistive diffusion is still incomplete for many classes of dynamos.

In the case of non-linear fast dynamos advances have been made through numerical magneto-hydrodynamical (MHD) simulations (e.g., Nordlund et al. 1992; Podvigina \& Pouquet 1994; Brandenburg et al. 1995; Zienicke et al. 1998; Archontis et al. 2003b; Schekochihin et al. 2004; Mininni et al. 2005b; Cameron \& Galloway 2006a). Although the details of the saturation mechanisms are unresolved it has been suggested that the dynamos saturate either because of a suppression of the stretching ability of the flows and a reduction of the field dissipation (Cattaneo et al. 1996; Archontis et al. 2003b, Tanner \& Hughes 2003) or via a balance between vigorous stretching and strong dissipation (Brandenburg et al. 1995).

In many astrophysical systems the stretching of the magnetic field lines, which drives the initial exponential amplification of the magnetic energy, is achieved by turbulent flows. An interesting issue is then the equilibrium field strength at which this turbulent dynamo action saturates. Moreover, if the generated magnetic field at saturation has magnetic energy equal to the kinetic energy of the flow (equipartition), then this dynamo is able to generate strong magnetic fields that are comparable with those observed in many astrophysical systems.

In this paper we present results from numerical MHD experiments with a non-helical dynamo a.k.a. the Archontis 
dynamo, as named by Cameron \& Galloway (2006a) after the work by Archontis (2000). The initial flow involved in these simulations is a non scale-separated flow, similar to the ABC flows but with no cosine terms. The dynamo action produced by this flow was first studied by Galloway \& Proctor (1992) and Galloway \& O'Brian (1993) in the linear, kinematic regime. These authors found that the growth rate of this dynamo increases as a function of $\mathrm{Rm}$ at least until $\mathrm{Rm}=800$ where the real part of the growth rate is close to 0.07 . The above growth rate was found for cases with non-randomly chosen initial magnetic field conditions. Later on, Dorch \& Archontis (2004) used a randomly oriented seed magnetic field and found that the growth rate of the fastest growing eigenmode is higher by an order of magnitude. More precisely, they found that the average growth rate of the fastest growing magnetic eigenmode is $\gamma \approx 0.5$ for an experiment with $\mathrm{Rm}=\mathrm{Re}=100$. Similar growth rates in the kinematic regime found for experiments with higher values of the Reynold numbers.

Archontis (2000) studied the non-linear dynamo action of this flow and found that the magnetic energy after the exponential growth saturates at equipartition with the kinetic energy in a laminar fashion for $\mathrm{Rm}=\mathrm{Re}=100$, where $\mathrm{Re}$ is the fluid Reynolds number $(\operatorname{Re}=U \ell / v, v$ being the viscosity). The ratio of the magnetic to the fluid Reynolds number is the magnetic Prandtl number $\left(\operatorname{Pr}_{\mathrm{m}}=\mathrm{Rm} / \mathrm{Re}\right)$.

Dorch \& Archontis (2004) performed a parameter study to examine the saturation level of the dynamo and they found that if $\operatorname{Pr}_{\mathrm{m}}$ is different from unity the velocity and magnetic fields are not identical but they are still proportional: e.g., for $\mathrm{Rm}=100$ and $\operatorname{Re}=200$ the two fields are inclined by more than 10 degrees in less than $2 \%$ of the computational volume and the saturation level deviates from exact equipartition with only fractions of a percent. They also showed that there is a critical value $\operatorname{Re}^{(\mathrm{c})}$ above which the dynamo saturates below equipartition. The critical value increases with decreasing magnetic resistivity and can therefore be quantified in terms of either Rm or $\operatorname{Pr}_{\mathrm{m}}$ : for $50 \leq \mathrm{Rm} \leq 200, \mathrm{Re}^{(\mathrm{c})}$ turns out to be in the range $250 \leq \operatorname{Re}^{(\mathrm{c})} \leq 350$.

Cameron \& Galloway (2006a) studied the equipartition solution for large values of Rm and they found that there is no evidence that the laminar behaviour at equipartition is not asymptotic and concluded that this dynamo yield fields strong enough to be astrophysically relevant. Cameron \& Galloway (2006b) extended their previous work on this dynamo and discovered new classes of dynamos, which saturate also at close equipartition. The forcing and flow in these dynamos are not so symmetric and, thus, one may argue that are more generic examples of equipartition dynamos.

The objective of the present paper is twofold: firstly, we identify the fastest growing magnetic eigenmode in the kinematic regime and examine how it changes when the flow is modified due to magnetic feedback in the dynamical regime. Secondly, we illustrate that the equipartition laminar mode is not a timeindependent solution, as it was found in previous experiments, but it suffers from transitions between turbulent and equipartition modes during the evolution of the system.

The paper is organized as follows: Sect. 2 contains the equations and the numerical method used for the simulations. The initial topology of the flow is described in Sect. 3. The amplification of the magnetic field and the subsequent saturation state for $\mathrm{Rm}=\mathrm{Re}=100$ are discussed in Sects. 4.1 and 4.2. Section 4.3 shows the behaviour of the dynamo for larger values of the Reynold numbers. The structures of the velocity, vorticity and magnetic field in physical space are visualized in Sect. 4.4. Section 5 contains the overall conclusions.

\section{Equations}

The full three-dimensional MHD equations are solved on a periodic Cartesian mesh using non-dimensional quantities;

$$
\begin{aligned}
\frac{\partial \boldsymbol{\rho}}{\partial t} & =-\nabla \cdot \rho \boldsymbol{u}, \\
\frac{\partial(\rho \boldsymbol{u})}{\partial t} & =-\nabla \cdot(\rho \boldsymbol{u} \boldsymbol{u})-\nabla P+\boldsymbol{j} \times \boldsymbol{B}+\boldsymbol{f}+\frac{1}{\mathrm{Re}} \nabla^{2} \rho \boldsymbol{u}, \\
\frac{\partial e}{\partial t} & =-\nabla \cdot(e \boldsymbol{u})-P \nabla \cdot \boldsymbol{u}+Q_{\mathrm{v}}+Q_{\mathrm{J}}+Q_{\mathrm{cool}}, \\
\frac{\partial \boldsymbol{B}}{\partial t} & =\nabla \times(\boldsymbol{u} \times \boldsymbol{B})+\frac{1}{\mathrm{Rm}} \nabla^{2} \boldsymbol{B} .
\end{aligned}
$$

We consider a fully compressible plasma with an energy equation (Eq. (3)) and equation of state to obtain the gas pressure. The gas pressure is $P=(\gamma-1) \rho \epsilon$, where $\epsilon$ is the internal energy $(e / \rho)$ per unit mass (or the specific internal energy). The ratio of specific heats, $\gamma$, is taken as $5 / 3$.

In Eqs. (1)-(4) above, $\rho$ is the fluid density, $\boldsymbol{u}$ is the velocity, $P$ the pressure, $\boldsymbol{j}$ is the electric current density, $\boldsymbol{B}$ is the magnetic field density and $e$ is the internal energy. In Eq. (2) $\boldsymbol{j} \times \boldsymbol{B}$ is the Lorentz force giving rise to the Lorentz work $W_{\mathrm{L}} \cdot Q_{\mathrm{v}}$ and $Q_{\mathrm{J}}$ are the viscous and Joule dissipation respectively. $Q_{\mathrm{cool}}$ is a Newtonian cooling term that balances the increase of the thermal energy due to the dissipative terms in the energy equation.

The external prescribed forcing term, $\boldsymbol{f}$, has the same form as the forcing we used in previous non-linear $\mathrm{ABC}$ dynamo experiments (Archontis et al. 2003b);

$f=-a(t) \frac{1}{\operatorname{Re}} \nabla^{2} \boldsymbol{u}$

We use again an amplitude factor in front of the driving force that is allowed to evolve with time in order to keep the average kinetic energy approximately constant in both laminar and turbulent phases during the action of the dynamo. The amplitude factor is given by

$a(t)=\frac{E_{0}}{E_{\mathrm{kin}}} e^{L}$,

where $L$ is determined from

$\frac{\mathrm{d} L}{\mathrm{~d} t}=\frac{1}{\tau} \ln \frac{E_{0}}{E_{\mathrm{kin}}}$

with $L=0$ at $t=0$. Equation (7) evolves the amplitude factor $e^{L}$ on the turn over time scale, $\tau$, if the kinetic energy starts to deviate from the initial value $E_{0}$. The factor $E_{0} / E_{\text {kin }}$ helps to reduce the time delay that results from the integral nature of the exponential factor in Eq. (6) and the delayed response of the kinetic energy on changes in the driving of the system. Thus, the amplitude of the forcing can compensate for the increased dissipation in the turbulent phase by increasing the forcing when the kinetic energy drops below $E_{0}$. We should also mention that, when turbulence kicks in (see the experiments in Sect. 4) the compressibility effects manifest themselves in the flow so that incompressible fluid flow analysis is no longer applicable in most of the volume of the flow field.

The above equations are solved numerically on a staggered mesh using derivatives and interpolations that are of 6th 
and 5th order respectively, in a numerical scheme that conserves $\nabla \cdot \boldsymbol{B}=0$ exactly. The time stepping uses a third order predictor-corrector method (Hyman 1972). Numerical solutions are obtained on a grid of typically $96^{3}$ points, using a modified version of the code by Nordlund and others (e.g. Nordlund et al. 1992).

\section{Flow topology}

The initial velocity field belongs to the well-known class of three-dimensional, steady $\mathrm{ABC}$ flows but does not contain cosines and is thus given by

$\boldsymbol{u}=(\sin z, \sin x, \sin y)$,

where the Cartesian coordinates $(x, y, z)$ are $2 \pi$-periodic. In contrast to the normal ABC flows, the initial vorticity $\omega=\nabla \times \boldsymbol{u}$ and the velocity field are not parallel and thus the kinetic helicity density $h=\boldsymbol{u} \cdot \omega$ has several local minima and maxima. However, the total kinetic helicity of this flow is equal to zero

$\int_{V} \boldsymbol{u} \cdot \omega \mathrm{d} V=0$.

Thus, although locally helicity density is not zero, there is no overall integrated helicity in the sytem.

The flow is divergence-free, very symmetric, with significant regions of chaotic particle trajectories and contains two different types of stagnation points. There are stagnation points where the streamlines are diverging in a two-dimensional plane with a spiraling form and are converging in an axis perpendicular to the plane (a-type), and stagnation points with the opposite topology of the streamlines (b-type). The diverging streamlines from one type of the stagnation points become converging for the other and vice versa.

Similarly to the velocity field, the vorticity has eight null points which are found in the middle of the straight heteroclinic connections of the stagnation points of the flow. In four of them the vorticity lines follow converging spiraling paths in a twodimensional plane and diverge along a perpendicular axis to the plane (b-type vortex null-points). The directions are reversed at the remaining four null points of the vorticity. Another interesting link between the velocity and the vorticity field is that the sites of maximum vorticity coincide with the b-type stagnation points of the velocity flow.

Figure 1 shows how stagnation points and vortex null points are connected along a main diagonal of the computational box. Streamlines coming from two a-type stagnation points (at the upper-right and at the lower-left corner) are converging in the center of the box where a two-dimensional spiralling unstable manifold exists. Null points of vorticity (marked as "a-null" and "b-null" in the figure) are located in the middle of the straight heteroclinic connections of the stagnation points. It is important to understand how the network of streamlines and vortex lines connect individual point with very small velocity and vorticity magnitude because the evolution of vorticity in association with the topology of the flow is a key process of the dynamo action.

\section{Results and discussion}

The following results are based on nonlinear experiments, which have been performed over many diffusive time scales using various values for the magnetic and fluid Reynolds numbers. Results from a wider range of parameters, but for a shorter time scale, have been presented in Dorch \& Archontis (2004). They showed

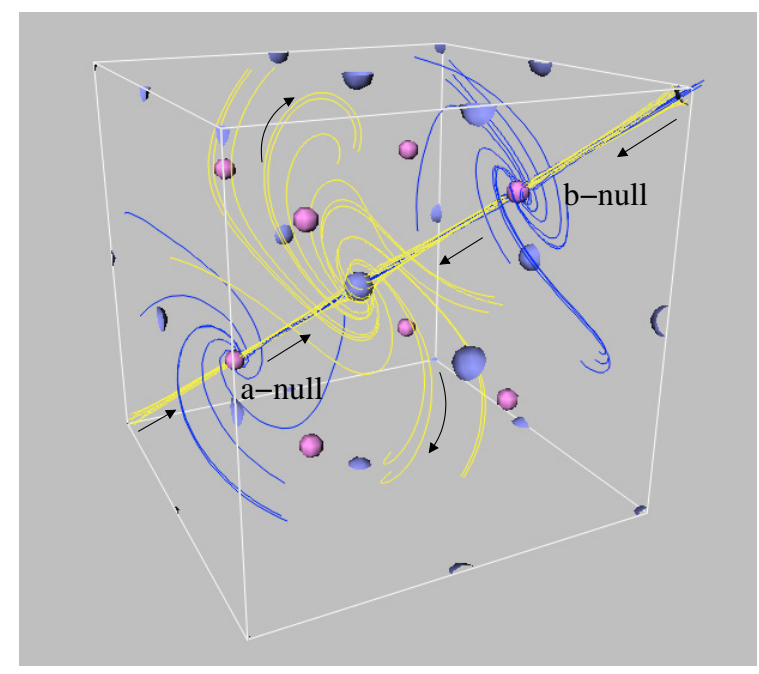

Fig. 1. Visualization of the stagnation points of the flow (blue) and the vortex null points (purple) and corresponding field lines (yellow for the velocity field and blue for the vorticity). The arrows show the sense of the velocity field vector.

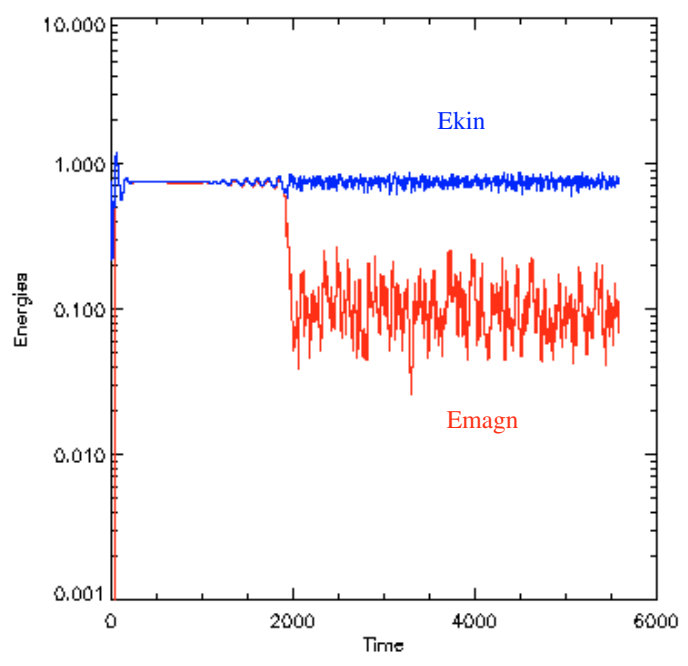

Fig. 2. Temporal evolution of the kinetic and magnetic energies for the experiment with $\mathrm{Rm}=\mathrm{Re}=100$.

that equipartition is achieved after saturation and that at sufficiently low $\operatorname{Pr}_{\mathrm{m}}$ (e.g., $\mathrm{Pr}_{\mathrm{m}}=0.16$ ) the dominating dynamo mode is in fact a decaying mode. In the following, we focus more on the experiments with larger $\operatorname{Pr}_{\mathrm{m}}$. In all experiments, we start with a random magnetic field, which first grows exponentially and then saturates in the dynamical regime.

\subsection{Fast dynamo action and saturation with $R m=R e=100$}

We first give results for the case with $\mathrm{Rm}=\mathrm{Re}=100$. The experiment has been performed at $64^{3}, 96^{3}$ and $128^{3}$ resolution producing similar results, concerning the temporal evolution of the energies and the spatial structures of the vector magnetic and velocity fields.

Figure 2 shows the growth and the saturation of the magnetic energy together with the time evolution of the kinetic energy for this experiment. At the beginning of the simulation, the magnetic energy increases exponentially with time and reaches equipartition values at $t \approx 43$. In the non-linear regime, there are three different phases in the evolution of the magnetic energy: first 


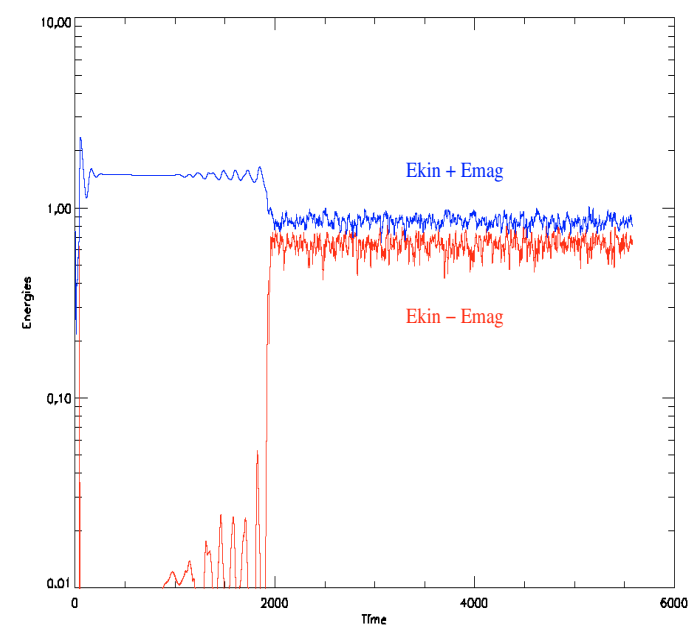

Fig. 3. Temporal evolution of the sum, $\left(E_{\mathrm{kin}}+E_{\mathrm{mag}}\right)$, and the difference, $\left(E_{\mathrm{kin}}-E_{\mathrm{mag}}\right)$, between the kinetic and magnetic energies.

an almost exact equipartition phase where the energies remain constant with a value of 0.75 up to time $t \approx 800$, then a phase, with a time-dependent solution, where the energies evolve with oscillations until $t=1900$ and then a fully developed turbulent phase where the magnetic energy drops by almost one order of magnitude and saturates with irregular fluctuations. In the last phase, the kinetic energy shows, similar to the magnetic energy, an episodic behaviour but stays close to its nominal value due to the action of the time-dependent forcing.

The time evolution of the sum and the difference between the energies, $E_{\text {sum }}=E_{\text {kin }}+E_{\text {mag }}$ and $E_{\text {dif }}=E_{\text {kin }}-E_{\text {mag }}$, is shown in Fig. 3. Cameron \& Galloway (2006a) showed that $E_{\text {sum }}$ and $E_{\text {dif }}$ evolve to a steady state and that the kinetic and magnetic energies show only small variations in time. They also calculated the cross helicity and found that the velocity flow and the magnetic field become, almost perfectly, aligned as the system evolves.

In our experiment (see Fig. 3), we find that the difference between the energies is very small until $t \approx 1900$, since the two energies have similar values. However, after $t=1900$ the magnetic energy drops and enters a turbulent regime. Thus, $E_{\text {dif }}$ increases rapidly by almost two orders of magnitude and later on saturates with fluctuations. Accordingly, the sum of the energies, $E_{\text {sum }}$, decreases and looses its time-independent nature, fluctuating around the value of 0.65 , until the end of the experiment.

We calculate the cross helicity to study the alignment of the two vector fields, similar to the previous work by Cameron \& Galloway (2006a). Figure 4 shows that the cross helicity has its maximum value, one, until $t \approx 1900$, indicating that the velocity and magnetic fields are parallel. However, at a later stage of the experiment turbulence appears and thus the cross helicity drops below its equilibrium value. The perfect alignment reported by Cameron \& Galloway (2006a) does not persist further. Now the two fields are not aligned making an average angle between 80 and 100 degrees during the evolution of the system.

It is useful now to calculate the average length scales of the fields in order to extract information about the dynamo behaviour in the non-linear and saturated phases. For the magnetic field, an average value of the length scale is given by

$L_{\mathrm{b}}=\left(\frac{\left\langle B^{2}\right\rangle}{\left\langle J^{2}\right\rangle}\right)^{1 / 2}$,

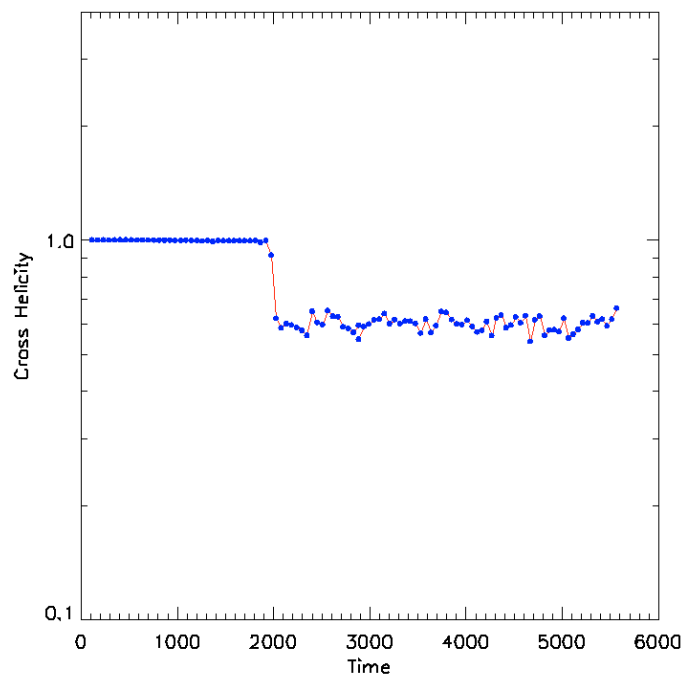

Fig. 4. Temporal evolution of the normalised cross helicity $\frac{\boldsymbol{U} \cdot \boldsymbol{B}}{(|\boldsymbol{U}|)(\boldsymbol{B} \mid)}$ for the experiment with $\mathrm{Rm}=\mathrm{Re}=100$.

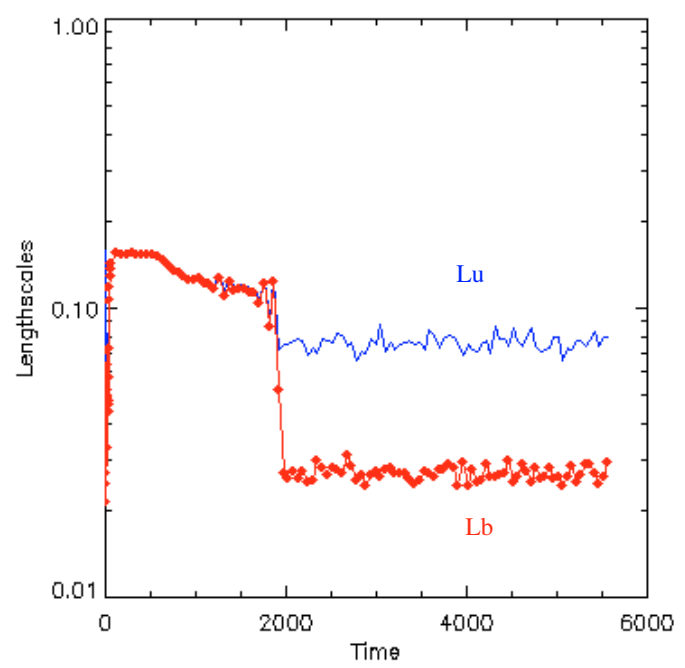

Fig. 5. Time evolution of the velocity lengthscale $\left(L_{\mathrm{u}}\right)$ and the magnetic lenghtscale $\left(L_{\mathrm{b}}\right)$.

where $J$ is the current. A measure of the length scale of the flow is given by

$L_{\mathrm{u}}=\left(\frac{\left\langle U^{2}\right\rangle}{\left\langle W^{2}\right\rangle}\right)^{1 / 2}$,

where $W$ is the vorticity.

Figure 5 shows the time evolution of the above length scales. In the kinematic regime of the dynamo, the length scales remain small. They start to increase when the magnetic energy becomes equal to the kinetic energy at time, $t \approx 43$. Eventually, they saturate (reaching a value of $\approx 0.15$ ) and become equal for a long period of time, until $t \approx 1900$. During equipartition, the average value of the lengthscales drops slightly. A rapid decrease occurs after turbulence (which has been identified due to Kolmogorov scaling in energy spectra) kicks in, with the lengthscale of the magnetic field to decrease by a factor of four and fluctuates around $L_{\mathrm{b}} \approx 0.03$ until the end of the simulation. The velocity field lengthscale experiences a less steep decrease, having a higher value $\left(L_{\mathrm{u}} \approx 0.8\right)$ than the lengthscale of the magnetic field in the turbulent phase. In any case, it appears that the fields have a smaller filling factor than in the first equipartition phase. 

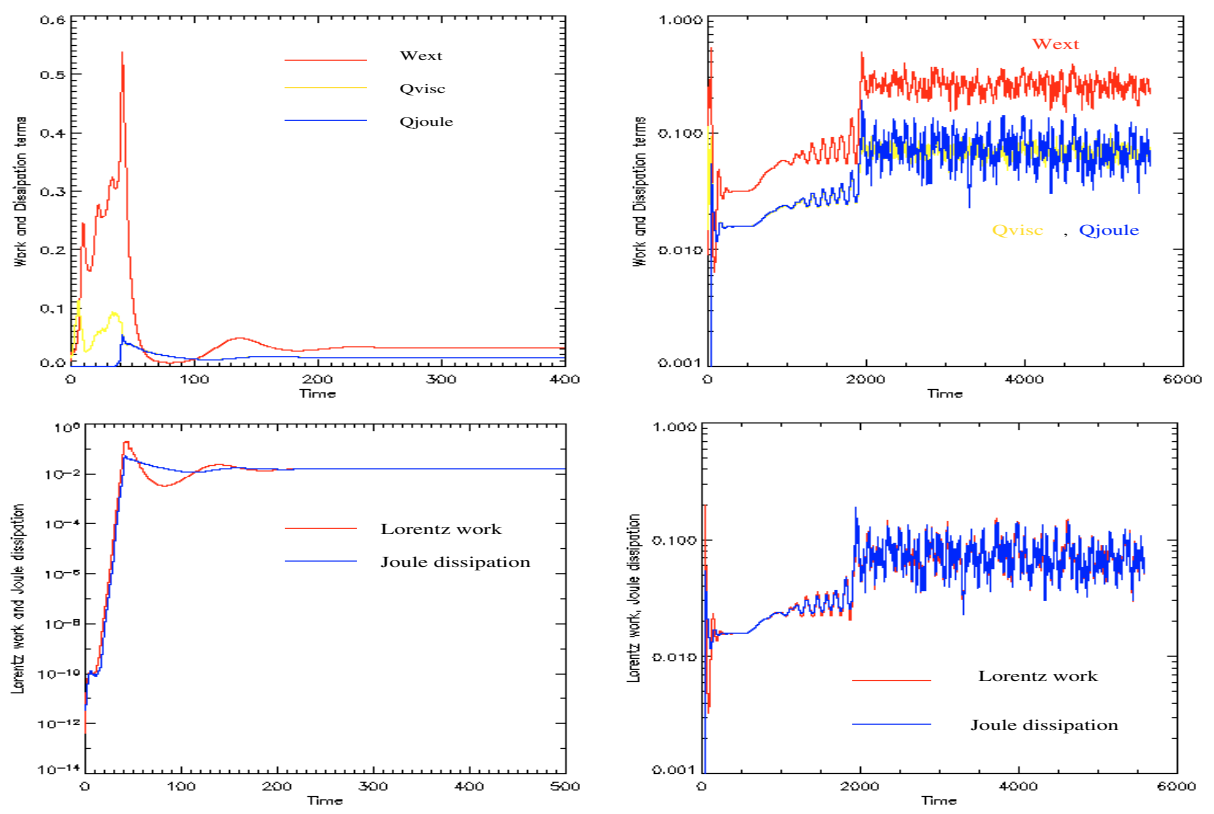

Fig. 6. Time evolution of the viscous and Joule dissipation $\left(Q_{\mathrm{v}}\right.$ and $Q_{\mathrm{J}}$ respectively) and the work done $\left(W_{\text {ext }}\right)$ by the prescribed external forcing for the experiment with $\mathrm{Rm}=\mathrm{Re}=$ 100 .

Thus, it seems that the length scale, on which the dynamo operate, of the two fields change considerably and deviate from each other just after the appearance of the turbulent phase. The velocity and magnetic fields become much less regular and the spatial scales decrease. Also, it is likely that since the scale at which the magnetic field varies decreases, the dissipation will increase as we discuss in the next section.

\subsection{Dissipation and work clues}

In this section, we consider an analysis of the work and dissipation terms, which are important for the understanding of the nature of the dynamo action in our experiments.

Firstly, we should mention that at the beginning of the simulation (until $t \approx 5$ ) the kinetic energy drops by up to about $50 \%$. This initial large decrease of the kinetic energy appeared also in the previous experiments by Dorch \& Archontis (2004) and Cameron \& Galloway (2006a). It is likely that this drop of the kinetic energy is due to viscous dissipation that occurs mainly near the sites of strong vorticity. The fluid develops local enhancements of vorticity where energy is dissipated more rapidly than elsewhere in the computational volume. Moreover, we find that the average vorticity and the viscous dissipation have similar temporal evolution during the simulation.

We will now examine the evolution of the work and dissipation terms during the simulation. Figure 6 consists of four frames and it shows the external work $W_{\text {ext }}$, the Lorentz work and the viscous and Joule dissipations as a function of time for this experiment. The left column shows the evolution of the terms at the early stages of the simulation, while the right column shows the entire evolution during the simulation.

The top-left frame shows that in the kinematic regime (up to $t \approx 40$ ) the work done by the external force $W_{\text {ext }}$ increases. As we discussed earlier, the kinetic energy drops at the beginning of the simulation but it is necessary to maintain the kinetic energy in order to ensure that the turn-over time and the actual fluid Reynolds number remain nearly constant during the experiment. Thus, the increase of the $W_{\text {ext }}$ is due to maintenance of the kinetic energy that slowly returns to its nominal value where at the beginning it is stabilized with no major fluctuations. The decrease of the kinetic energy in the kinematic regime is much smaller when the fluid Reynolds number has a lower value (in the latter case, the velocity field remains almost stationary with no particular deviations from its original configuration). The viscous dissipation follows the oscillatory growth of the external work in the kinematic regime and their difference $\left(W_{\text {ext }}-Q_{\mathrm{v}}\right)$ fluctuates around zero.

When the magnetic field becomes strong enough and begins to modify the flow through the Lorentz force (after $t \approx 30$ ), the external work increases rapidly, and almost monotonically with time, reaching its highest value at $t \approx 40$. Joule dissipation and Lorentz work also have local maxima at $t \approx 40$ and saturate soon thereafter. The above mentioned time is the first instant of energy equipartition, although the kinetic energy has not yet returned to its nominal level.

The same frame also shows that in the early saturation regime viscous and Joule dissipation get a constant value, each being equal to half of the rate of work done by the external force so that:

$Q_{\mathrm{v}}=Q_{\mathrm{J}}=W_{\mathrm{ext}} / 2$.

The bottom-left panel shows that the average growth of the magnetic energy in the kinematic regime is accompanied by a small difference between the work done against the Lorentz force $W_{\mathrm{L}}$ and the Joule dissipation $Q_{\mathrm{J}}$. This small positive difference, and by analogy with previous experiments (see Archontis et al. 2003b and Dorch \& Archontis 2004), occurs in the regions where the magnetic field and the dissipation is very weak. These regions cover most of the computational volume and almost all of the net work (defined by the difference $W_{\mathrm{L}}-Q_{\mathrm{J}}$ ) occurs there. Joule dissipation is strong close to the structures with high magnetic field strength, but is balanced by the work done on the field by the flow through advection and convergence. In other words, the exponential amplification of the magnetic energy is due to the stretching of the weak magnetic field in the bulk of the background flow. Although the strong magnetic field structures occupy a small percentage of the volume, they do not play an important role for the fast growth rate of the dynamo.

After the onset of saturation, there is a small transient phase and later on the two terms become equal with their evolution being time-independent until $t \approx 500$. The above analysis of the work and dissipation terms, shows that the saturated dynamo 


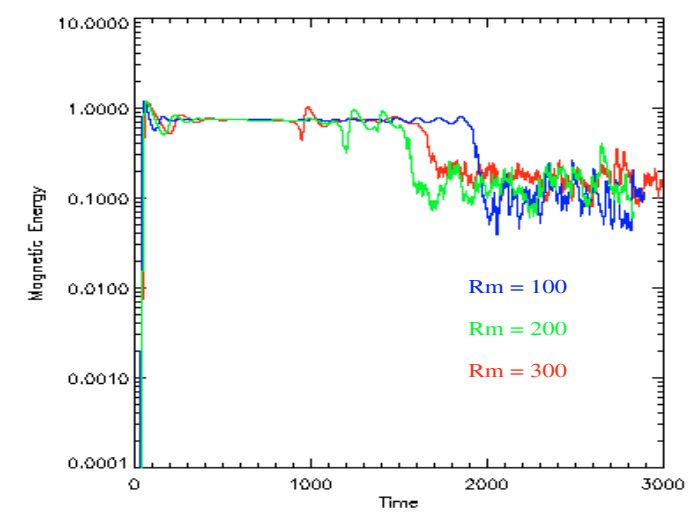

Fig. 7. Time evolution of the magnetic energies for the experiments with $\mathrm{Re}=100$ and $\mathrm{Rm}=100,200$ and 300 .

stays at equipartition and also does not grow or decay, since the sum of the resistive and viscous losses is balanced exactly by the work terms.

Thus the dynamo, after it goes through a kinematic phase, comes out as a laminar solution with the magnetic field being equal in direction and magnitude to the velocity field. Then, one may show analytically that this is only an exact solution to the MHD equations in the absence of forcing and dissipation. As an example, for $\operatorname{Pr}_{\mathrm{m}}=1$, the diffusion terms are both proportional to the velocity and magnetic field strength, respectively, with the same constant of proportionality being $1 / \operatorname{Re}$ (while one can assume that $\rho \approx 1$ and $\nabla P \approx 0$ ).

Now, a striking result is that magnetic and kinetic energies do not remain equal until the end of the simulation and, eventually, the equipartition breaks down. The top-right panel in Fig. 6 shows that the external work and the dissipation terms increase slowly with oscillations between $t=500$ and $t=1900$ but then they experience a much more pronounced increase, which is followed by irregular fluctuations. In general, the increase of the external work is due to the deviation of the velocity field from its initial configuration. In a similar manner, the viscous and Joule dissipation increase, especially when the flow enters the fully developed turbulent state at around $t=1900$. The bottom-right panel shows another interesting result. The detailed balance between the Joule dissipation and the Lorentz work exists throughout the simulation even if the flow becomes turbulent and the equipartition breaks down. The small difference between the two terms in the turbulent phase becomes positive when the magnetic energy grows and negative when the magnetic energy decays.

\subsection{Behaviour with large values of $\mathrm{Rm}$ and $\mathrm{Re}$}

In the previous section we studied the case with $\mathrm{Rm}=\mathrm{Re}=100$. Now we present results from experiments with larger values of the Reynold numbers. In all cases we use the same forcing and start with the same random initial configuration for the magnetic field, as in our first experiment.

Figure 7 shows the time evolution of the magnetic energy for experiments with $\operatorname{Pr}_{\mathrm{m}}=1$ and increasing values of $\mathrm{Rm}$. When $\mathrm{Rm}=200$ and $\mathrm{Rm}=300$ the system evolves first to a steady state, similarly to the $\mathrm{Rm}=100$ experiment, up to $t \approx 900$. Then, a second time-independent phase begins with fluctuations, which are relatively larger from the $\mathrm{Rm}=100$ experiment, in the magnetic energy. Finally, magnetic energy drops steeply at around $t=1400$, earlier than in the $\mathrm{Rm}=100$ case, and the system follows a turbulent state. Thus, it seems that turbulence kicks in faster in the experiments with larger Reynolds number, although the onset of the turbulent phase appears at similar times for the cases with $\mathrm{Rm}=200$ and 300 . Also, the saturation level of the magnetic energy is similar between the experiments.

The top panels in Fig. 8 show the evolution of the kinetic and magnetic energies for the two experiments $(\mathrm{Rm}=\mathrm{Re}=200$ and $\mathrm{Rm}=\mathrm{Re}=300$ ). At the beginning of the saturation phase the magnetic energy reaches almost perfect equipartition with the kinetic energy. A very interesting result now is that in the second phase, which is a time-independent phase, the two energies remain close together except from small time intervals (for example, at $t=1100$ for the $\mathrm{Rm}=200$ experiment and at $t=900$ for the $\mathrm{Rm}=300$ case) where the magnetic energy decreases and deviates temporarily from the kinetic energy. In the last turbulent phase, the kinetic energy shows random fluctuations with small amplitude and stays close to its nominal value.

The results of two more experiments, one with $\mathrm{Rm}=200$ and $\mathrm{Re}=100$ and the other with $\mathrm{Rm}=400$ and $\mathrm{Re}=100$, are shown in the bottom panels in Fig. 8. The general evolution of the system is similar to the previous experiments (see top panels in the same figure). However, there are also some differences between the experiments. The oscillations in the energies, before the fully developed turbulent state, have large amplitudes and they exist over a long time period for the Rm $=400$ experiment. Notice, that although the solution becomes unstable the two energies follow a similar time evolution, which deviates from equipartition, mostly close to the minima of the oscillatory state during saturation. A striking result in these experiments is that the magnetic energy goes through a turbulent phase (after $t=1500$ ) but later on comes out as a laminar equipartition solution for short periods of time that are different in each experiment. However, the solution does not stay at equipartition but eventually becomes turbulent again. This transition of the magnetic energy between the two states is repeatedly observed during the experiments and it shows the very complex and timedependent evolution of the fields during the saturation of the system.

\subsection{Visualizing the dynamo modes}

Further features associated with the dynamo action and the equilibration of the magnetic field may be identified using threedimensional visualization. Thus, in this section we report on the behavior of the magnetic field, vorticity field and velocity flow in physical space. We consider first a case with very small fluid Reynolds number, the reason being that the flow becomes unstable for values of Re greater than eight. Thus, very small values of the fluid Reynolds number ensure that the flow remains smooth and stable during the exponential growth of the magnetic energy and we can follow closely the time evolution of individual structures in space. Then, we visualize the structures at saturation, developed in the experiments with higher values of $\mathrm{Re}$ and $\mathrm{Rm}$.

\subsection{1. $R m=100, R e=4$}

The time evolution of the energies in this case is similar to the previous experiments with higher values of Re and Rm: the fast growth in the kinematic regime is followed by a saturation of the magnetic energy at a level very close to equipartition, with $E_{\mathrm{kin}} / E_{\mathrm{mag}} \approx 1.01$. It is worthwhile mentioning that the solution does not depart from the equipartition state until the end of the experiment at $t \approx 3500$, which is quite advanced in time in comparison with the other experiments. The reason for this long-lived time-independent solution is probably the low value 

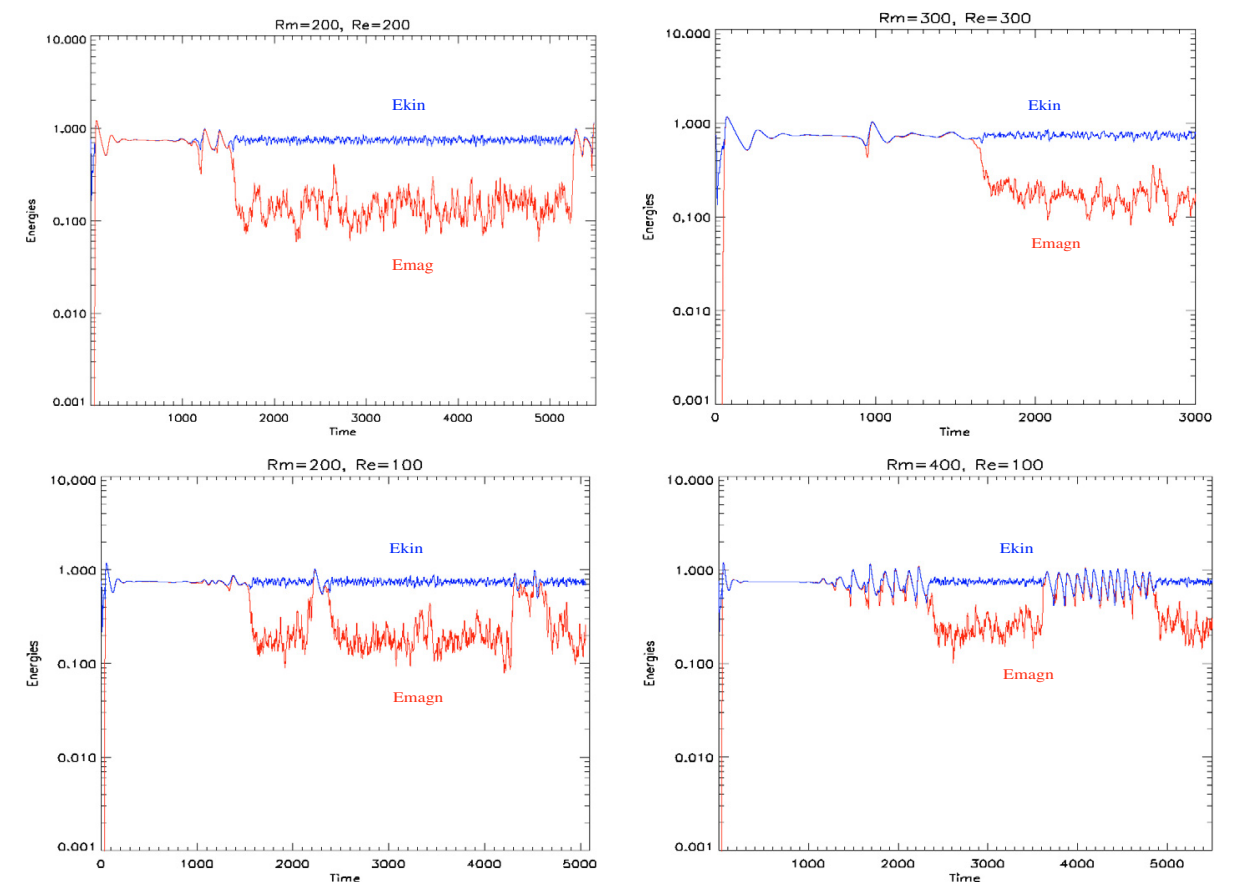

Fig. 8. Time evolution of the kinetic and magnetic energies in experiments with various values of Rm and Re.

of Re and the fact that this case is probably not optimum for the development of turbulence.

Figure 9 consists of three panels that show the topology of the structures in the kinematic regime. More precisely, the top panel shows that the weak velocity field adopts tube-like shapes with low gas pressure at the two-dimensional stable manifolds. The a-type stagnation points undergo only slight perturbation and are connected with the low pressure velocity tubes as follows: twisted streamlines, which are converging in twodimensional planes perpendicular to the tubes' axes, diverge along the axes of the tubes and towards the a-type stagnation points. After their ejection from the a-type stagnation points the streamlines continue into the neighboring tube-like regions of weak velocity, where they again follow spiral converging paths and vice versa.

Now we would like to emphasize that a key ingredient for understanding how the dynamo works in the kinematic regime is the evolution of the vorticity field, which is spatially related to the strong magnetic field structures. At the beginning of the simulation (up to $t=5$ ) the average vorticity (and the viscous dissipation) increases rapidly. More precisely, vorticity is rapidly advected in the vicinity of the initial b-type stagnation points of the flow and forms eight strong vortex tubes that are connected with the low pressure regions at their ends. On the other hand, the vortex null points move along the heteroclinic connections and approach the a-type stagnation points of the flow. As an example, the two null points that are located in the diagonal of the computational box shown in Fig. 1 come closer to the central atype stagnation point of the flow. After this initial transient phase the average vorticity increases slowly and the size of the strong vortex tubes changes slightly.

The middle panel in Fig. 9 is a visualization of the vorticity. Looking at the vorticity lines we find that "three-fold-like" symmetries have now been established between the strong vortex tubes and the vortex null points. Three vortex tubes (marked as "A", "B" and "C") form a triangle and another vortex tube (marked as " $\mathrm{D}$ " in the figure) is located on the axis that is passing through the center of the triangle. A null point is located exactly there, at the center of the triangle. If the null point is of the "a-type" the vorticity lines diverge from the three vortex tubes and they converge to the null point. They then diverge again along the axis and continue to the fourth vortex tube. If the null point is of "b-type" the direction of the vorticity lines is the opposite. Note that the two null points are connected (through a btype stagnation point) and thus the vorticity lines are not trapped inside each sub-volume that contains a "three-fold" symmetry, but they are instead piled up towards the vortex tubes that they find in their way. It is worthwhile to mention that the existence of "three-fold" symmetries for the vorticity field has not been reported before for this dynamo. Because of these symmetries the vorticity lines are stretched, twisted and folded in a similar way to the magnetic field lines in the normal ABC dynamo. In the latter case the operation of the fastest growing magnetic eigenmode occurs on the basis of a very symmetric "three-fold" situation and through a stretch-twist-fold and reconnection mechanism between “double-cigar” structures (Dorch 2000).

The magnetic field has the form of strong and curved sheets that are wrapped around the strong vorticity structures. The field lines from these strong sheets are stretched and twisted by the flow and finally pile up to neighboring sheets with similar polarity (see Fig. 9, bottom panel). In fact, most of the work done against the Lorentz force occurs between the strong flux sheets and the strong velocity field which surrounds them. The weak magnetic field has the form of rounded sheets that are folded at the a-type stagnation points. The folding of magnetic field lines in the plane of divergence of an a-type stagnation point is shown in Fig. 9 (bottom panel). Constructive folding of the magnetic field also occurs close to the center of the weak velocity channels. There, the magnetic field lines are folded in the same way as in the a-type stagnation points.

An interesting question now is what happens when the exponential amplification of the magnetic energy stops and the strength of the magnetic field becomes large enough for it to react back on the flow. The resulting Lorentz force on the fluid is perpendicular to the magnetic field and acts in the vertical direction. Moreover, it is found that the regions of maximum Lorentz force are correlated with high amplitude vorticity regions and the force acts typically perpendicular to the vorticity. When the 

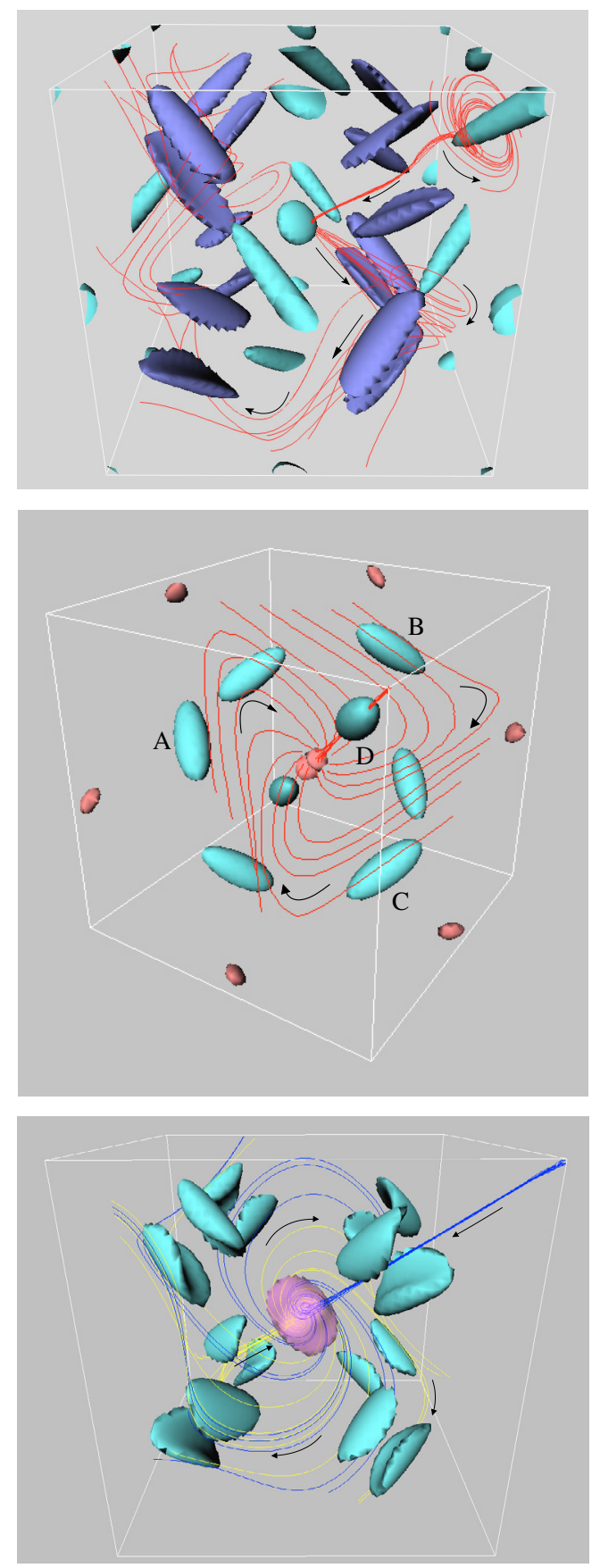

Fig. 9. Top: visualization of strong (weak) magnetic (velocity) structures (dark and light isosurfaces correspondingly) in the kinematic regime. Streamlines (coming from the up-right corner) converge first to an a-type stagnation point (center of the box), and then diverge and are twisted around weak velocity tubes as soon as they reach their neighbourhood. Middle: strong vorticity isosurfaces, null vortex points and vortex lines. Bottom: weak magnetic field (visualized only in the center of the box as a transparent isosurface), strong magnetic sheets and magnetic field lines. Most of the field lines are folded at the center of the box and then add to the neighboring sheets. In all figures, the arrows show the direction of the lines.

Lorentz force becomes strong enough the vortex strength in the high vorticity regions is reduced and the elongated vortex tubes shrink together with the velocity channels of low pressure that feed them. In fact, they retreat towards the initial position of the b-type stagnation points of the flow. The vorticity now between the magnetic flux sheets becomes very low and it almost adapts the properties of an a-type vortex null-point. Thus, it seems that the dynamical coupling of the vorticity and the magnetic field, which has not been reported in previous studies of this dynamo, is of potential importance to a better understanding of the magnetic energy amplification and the subsequent saturation.

The velocity field is also modified. Figure 10 is a first detailed and simultaneous visualization of the evolution of the magnetic, velocity and vorticity field. Such animations help us to understand better the coupling between the fields and the transition from the kinematic to the saturation regime. More precisely, Fig. 10 shows that the strong velocity field, which was surrounding the magnetic flux sheets in the kinematic regime, is now accumulated inside the "voids" in between the strong magnetic structures and takes the form of roundish blobs. These "voids" are the sites of the newly formed a-type vorticity null points mentioned above. In the absence of the strong vortex tubes the magnetic sheets stop the spiral motion around them and are folded. In fact, the folding of the strong magnetic field ensuing the accumulation of the high velocity field at the a-type null points of vorticity and leads to the formation of blob-like large scale structures. The two fields now (magnetic and velocity) are aligned in most of the computational domain.

It is of great importance to find where in the computational domain the relative orientation of the magnetic and velocity field is such that dynamo stops or still works effectively. Nearly perfect alignment - and thus no stretching of the magnetic field lines by the flow - is achieved where the two fields are strong. Joule dissipation is also at minimum there and thus the size and the strength of the relevant structures remain unchanged in time. The sites of imperfect alignment are the a-type stagnation points of the flow and their close vicinity. The work done against the Lorentz force at these sites is balanced by high Joule dissipation. Thus, a total balance between work and dissipation is achieved in most of the volume. The total kinetic and magnetic energies become equal and this solution tends to relax to a unique timeindependent state.

Another interesting question is how the magnetic field looks like when the magnetic energy saturates and where the magnetic field lines go and come from. Figure 11 shows the threedimensional structure of the strong magnetic field once dynamo approaches equilibration. The spiraling motion of the twisted sheets around the eight points of strong velocity and minimum vorticity is accompanied by the folding of these sheets and the formation of a coherent blob-like magnetic structure. Figure 11 also shows how the magnetic field lines establish links between the strong magnetic field structures and the stagnation points. The field lines shown in the figure are traced from the upperright corner of the numerical domain. They go through the folding area and as soon as they reach the center (where the magnetic field is weak and coincides with an a-type stagnation of the velocity flow) diverge in a spiral manner. After they are stretched they pile up to the magnetic structures at the b-type stagnation points of the flow. The magnetic field structures and the topology of the field lines remain in an approximately steady-state situation between $t \approx 83$ (see bottom-right panel of Fig. 11) and the end of the experiment at $t \approx 3500$.

\subsection{2. $\mathrm{Rm}=\mathrm{Re}=100$}

We have shown that the temporal evolution of the energies in this experiment (Sect. 4.1) follows first an equipartition and then a turbulent phase during saturation. The equipartition phase was also reported in the work by Cameron \& Galloway (2006a) who used the Elsässer variables, to show the source of the variation 

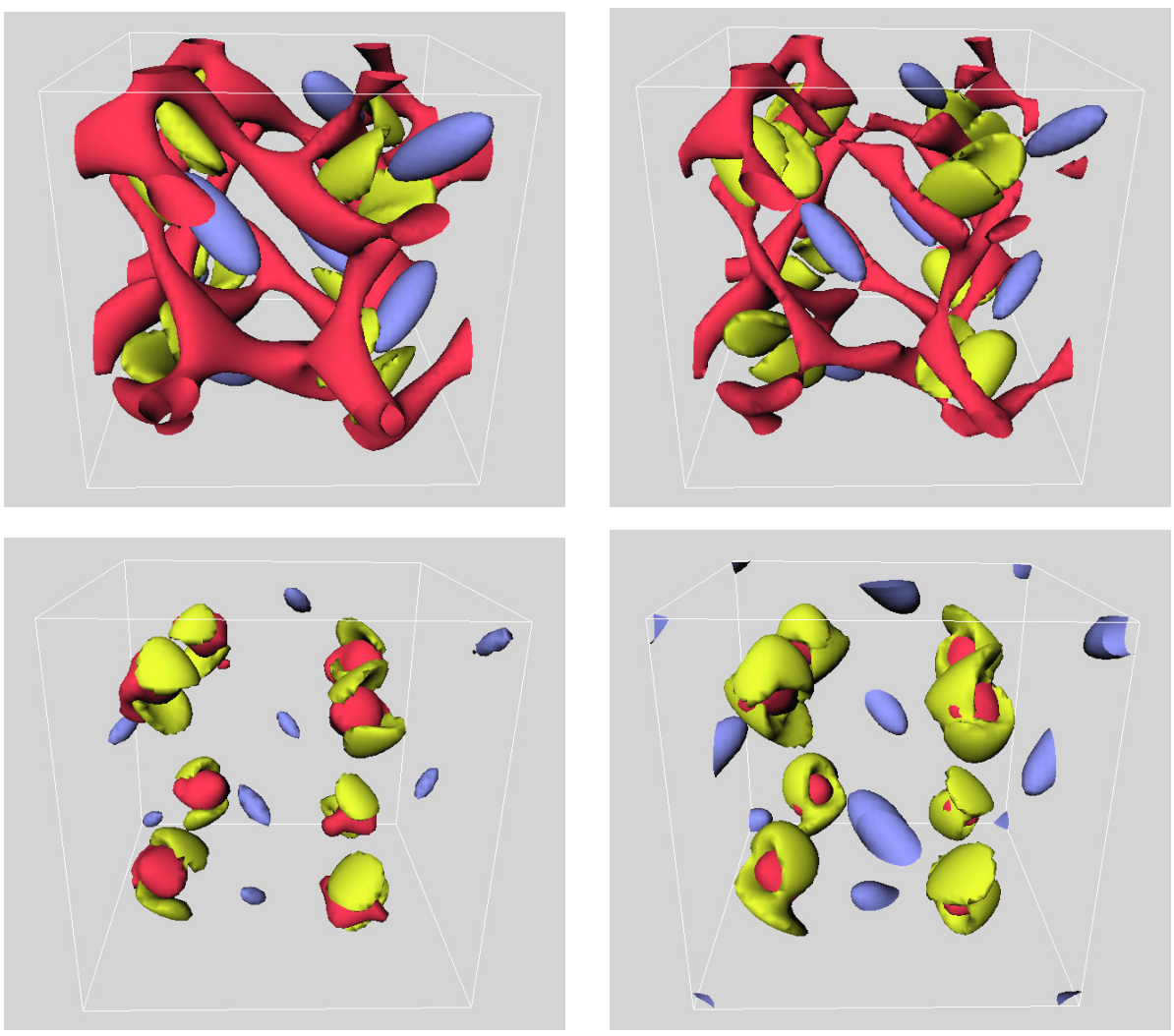

Fig. 10. Visualization of strong field structures (blue is the vorticity, red is the velocity and yellow is the magnetic field) just before saturation. Time increases from the top-left snapshot to the bottom-right one as follows: $t=26.4,27.5,28.7$ and $t=29.9$ respectively.
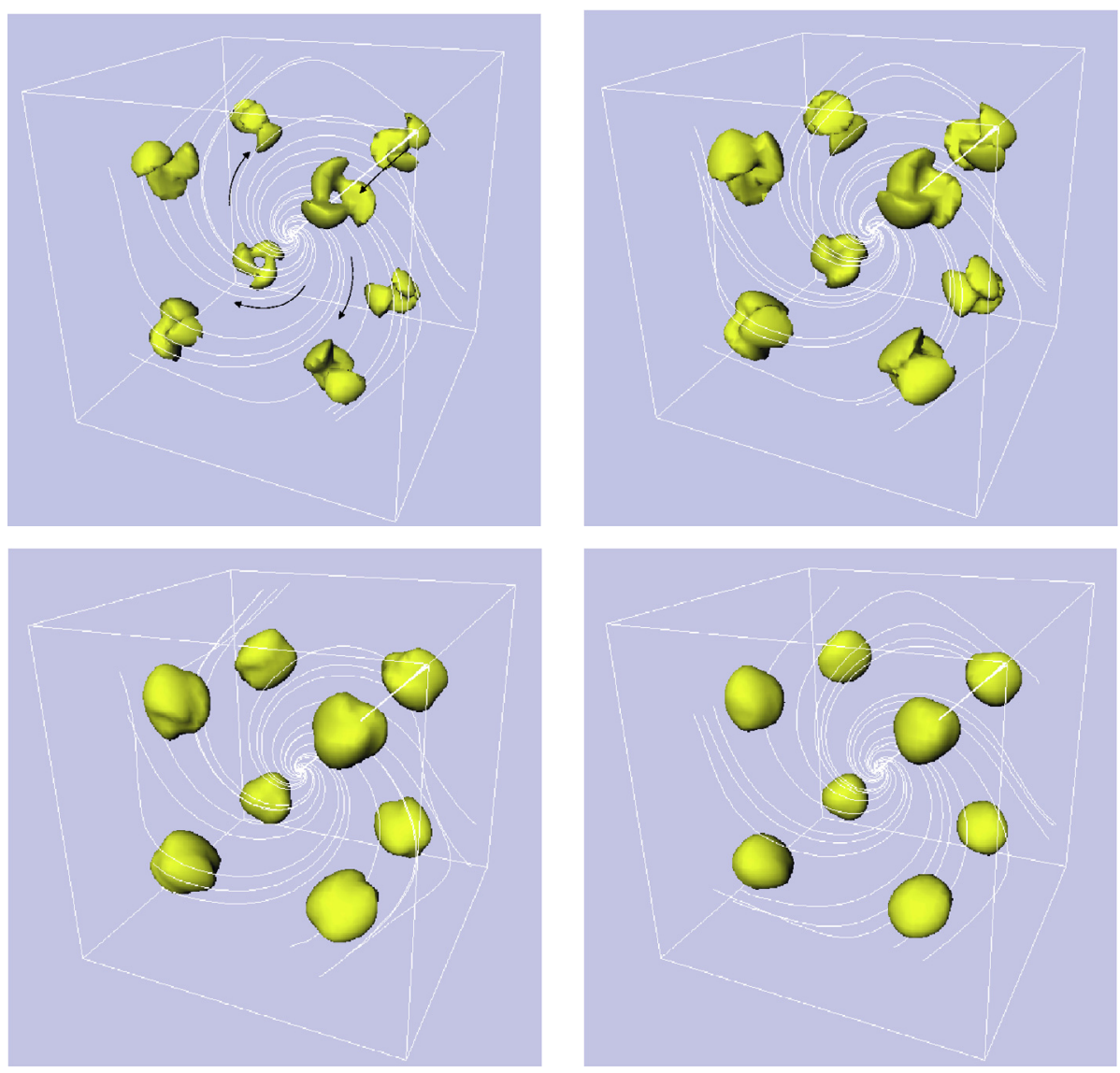

Fig. 11. Visualization of the magnetic field strength. The isosurfaces correspond to $85 \%$ of the maximum field strength in each snapshot. Shown is the spiral folding of the sheet-like structures and the creation of large-scale magnetic blobs. The lines are magnetic field lines and the arrows show their direction. The time for each snapshot (from top-left to bottom-right) is $t=75.1,76.6,81.3$ and $t=82.8$, respectively.

in the energies for the $\boldsymbol{U}=\boldsymbol{B}$ solution of this dynamo. They found that $(\boldsymbol{U}+\boldsymbol{B})$ is large and time-independent and, thus, most of the variations between the velocity and magnetic fields comes from $(\boldsymbol{U}-\boldsymbol{B})$. In this section, we visualize the structure of the
Elsässer variables in the two phases and study whether they are informative for the evolution of the system.

Figure 12 reveals $(\boldsymbol{U}+\boldsymbol{B})$ (top panel) and $(\boldsymbol{U}-\boldsymbol{B})$ (bottom panel) in the first energy-equipartition phase, at $t=139$. The 

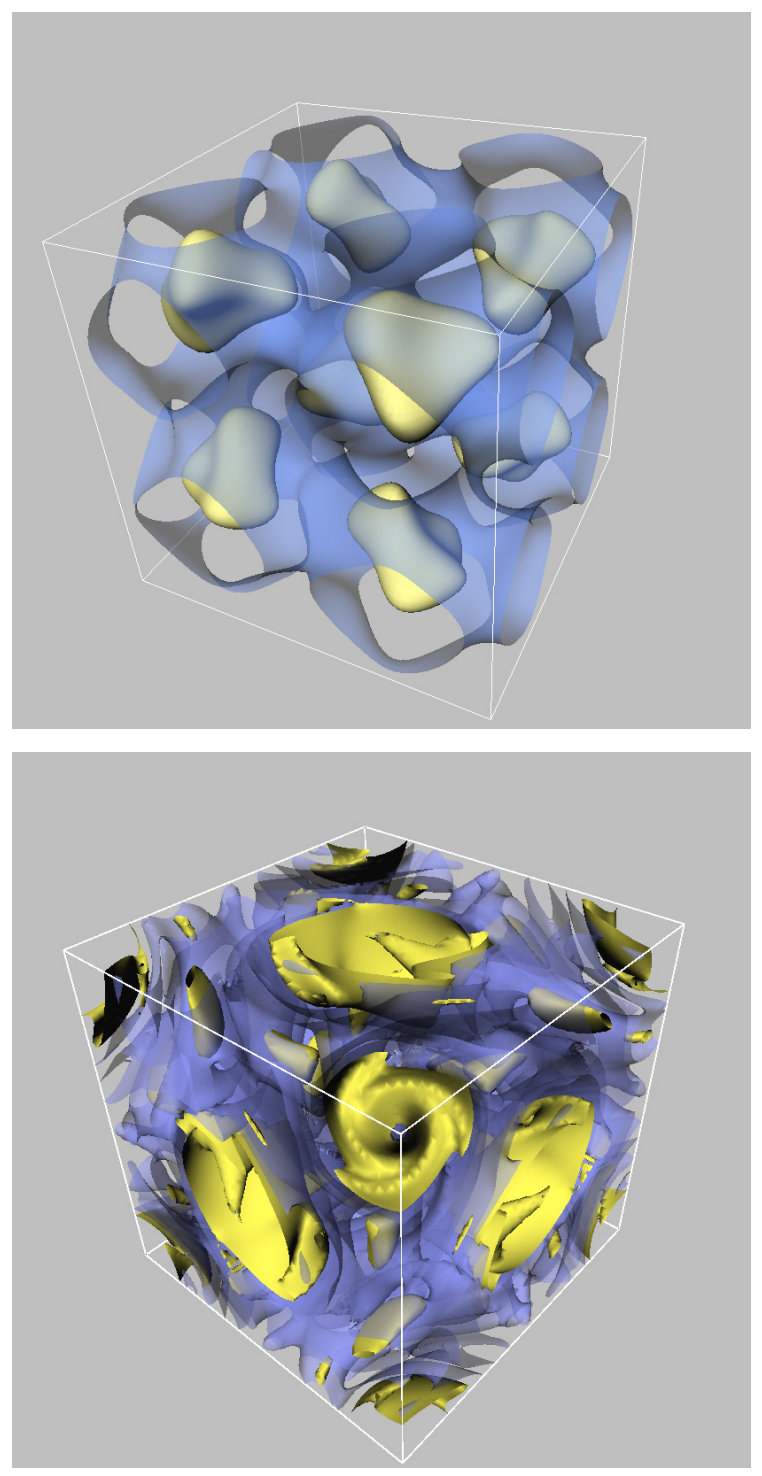

Fig. 12. Top: $3 \mathrm{D}$ visualization of the Elsässer variable $\boldsymbol{U}+\boldsymbol{B}$ for the case $\mathrm{Rm}=\mathrm{Re}=100$ when the magnetic energy is at equipartition with the kinetic energy. Bottom: the same as above for the $\boldsymbol{U}-\boldsymbol{B}$ variable.

structures of the Elsässer variables in these panels are similar to the structures shown by Cameron \& Galloway (2006a). The blue transparent isosurface in the top panel is 75 per cent of the maximum value of the $(\boldsymbol{U}+\boldsymbol{B})$ solution, while the yellow isosurface is close to 95 per cent. In fact, this yellow isosurface is reminiscent of the isosurface in Fig. 11 that shows folding of strong magnetic field structures. In the bottom panel, the yellow isosurface is about 50 per cent of the maximum value of $(\boldsymbol{U}-\boldsymbol{B})$ in this snapshot. Most of the surface is concentrated at the edges of the sides of the computational volume and adopts a spiral structure. In the middle of the spirals, there are weak tube-like structures which are oriented along the heteroclinic lines. The blue isosurface is only 15 per cent of the maximum value and has the form of flux sheets and ribbons, which fill up the volume in between the spiral structures.

Cameron \& Galloway (2006a) reported that is likely that the smooth structures of $\boldsymbol{U}+\boldsymbol{B}$ are less informative than the intermittent isosurfaces of $\boldsymbol{U}-\boldsymbol{B}$, which may provide a wealth of information for the saturation process and the equipartition of the energies. However, we find that a new and very interesting linkage is created between the Elsässer variables during equilibrium in the first phase of saturation, and thus, both variables are important. The top panel in Fig. 13 shows the yellow isosurface, which is 80 per cent of the maximum value of $\boldsymbol{U}-\boldsymbol{B}$, and the blue isosurface that is 10 per cent of the maximum value of $\boldsymbol{U}+\boldsymbol{B}$ at $t=868$. The yellow structures adopt a tube-like shape in most of the volume, although there are also sites where $\boldsymbol{U}-\boldsymbol{B}$ forms curved sheets (close to the center of the box). On the other hand, the structures with small values of the difference between the field vectors are concentrated in between the strong tube and sheet-like structures and connect them at their ends. It is worthwhile to mention that the small $\boldsymbol{U}+\boldsymbol{B}$ values coincide, in space, with the minima of cross helicity, which is a measurement of the alignment between the velocity and magnetic fields. Thus, it seems that most of the work done (although it is small due to equipartition) in the saturation regime occurs at the places with small values of $\boldsymbol{U}+\boldsymbol{B}$. In fact, visualization of magnetic fieldlines (red lines in the computational volume) show that significant twist and folding occurs exactly there. The fieldlines are running in parallel and are added to the tubes and sheets, but they are following highly twisted spiral paths when they approach the minima of $\boldsymbol{U}+\boldsymbol{B}$. Eventually, they are folded with fieldlines which are coming from neighboring structures and have similar polarity. This process increases the magnetic energy of the system. The bottom panel in Fig. 13 shows the same structures at $t=2618$, after the onset of turbulence. It seems that the variables have lost their smooth topology adopting irregular shapes. There is no clear connection between them and it is difficult to know if there is valuable information, which is locked up in the turbulent structure of these variables.

\subsection{3. $\mathrm{Rm}=400, \mathrm{Re}=100$}

In Sect. 4.3 we saw that the magnetic energy in the experiment with $\mathrm{Rm}=400$ and $\mathrm{Re}=100$ jumps from a turbulent state to another regime where it evolves with less-irregular oscillations at close equipartition with the kinetic energy. Thus, it is interesting to visualize the structure of the magnetic field when the magnetic energy has escaped from the turbulent phase and returns to the energy equipartition level.

Figure 14 shows the three-dimensional topology of the full magnetic field vector at two times: $t=3861$ (top panel) and $t=3888$ (bottom panel). These two snapshots have been chosen because the magnetic energy goes from a minimum (at $t=3861$ ) to a maximum value (at $t=3888$ ) and, thus, we may extract some information on how this mode operates, increasing the magnetic energy. Shown in the snapshots is the isosurfaces of $\boldsymbol{B}$ with a value of 70 per cent of the maximum.

The top panel shows that when the magnetic energy is minimum the field is concentrated into small-scale coherent tubes and sheets. This intermittent topology of the field is different to the topology at the first equipartition phase where the magnetic structures are smooth and adopt the shape of blobs, which are formed when the magnetic field is packed at special locations of the flow. The bottom panel shows that, later on, most of the structures are folded constructively in various positions inside the computational volume. For example, in the closest lower and the upper corner of the box in Fig. 14, large scale structures are formed due to twisting and folding of many small scale magnetic field tubes and sheets. The fieldlines adopt a spiral shape at the sites of folding, indicating the twisting motion of the structures prior to the folding that increases the magnetic energy of the system. The following decrease of the magnetic energy is accompanied by less twist and folding of the magnetic structures, 

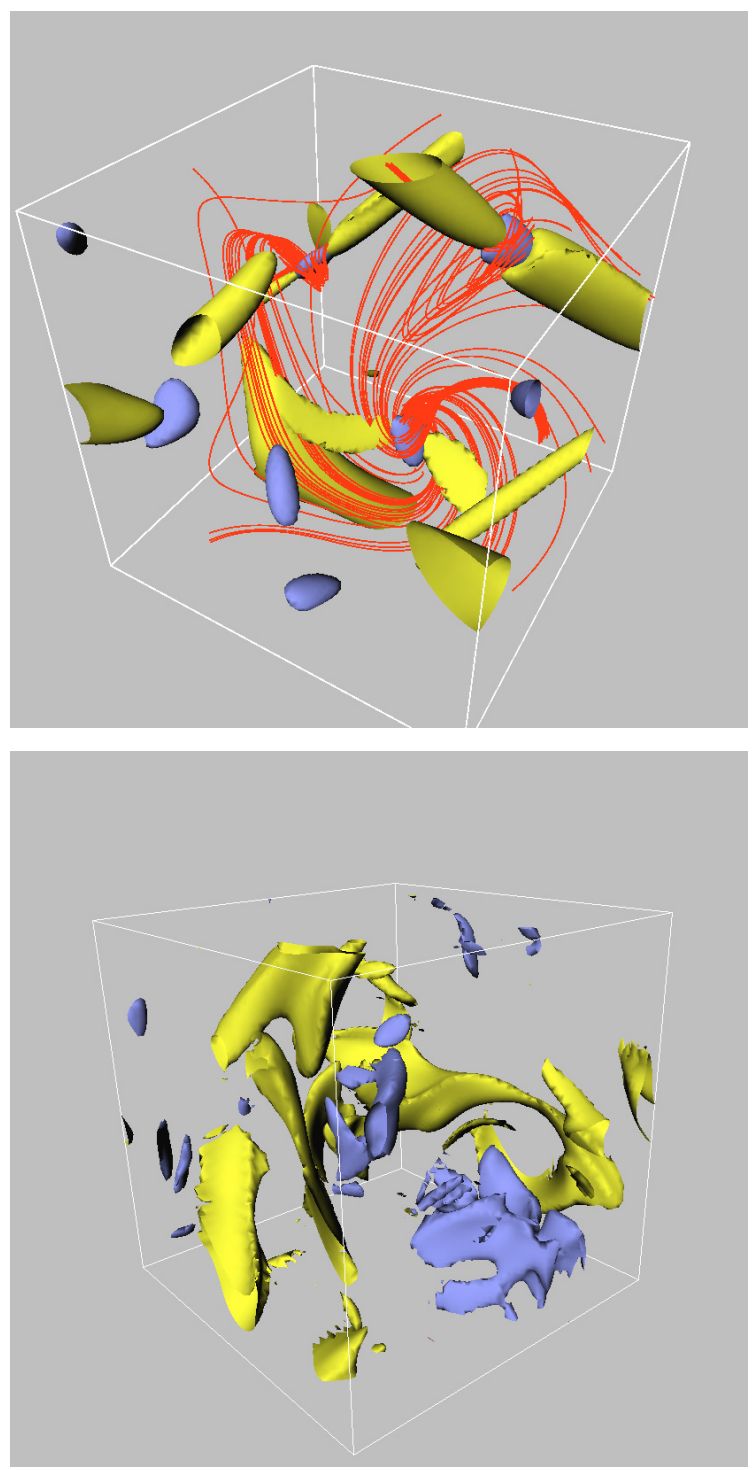

Fig. 13. Top: 3D visualization of isosurfaces with large values of $\boldsymbol{U}-\boldsymbol{B}$ and small values of $\boldsymbol{U}+\boldsymbol{B}$ during equipartition, at $t=868$. Bottom: the same structures in the turbulent regime of the saturation.

which eventually take again the form of individual tubes and sheets. We assume that the same physical processes occur when the magnetic energy decays or grows during the temporal evolution of the system in this phase of saturation.

\section{Conclusions}

This paper presents results from numerical MHD experiments of non-helical and nonlinear compressible fast dynamo action while varying the characteristic dimensionless parameters $\mathrm{Rm}$ and $\operatorname{Re}$ (and hence $\operatorname{Pr}_{\mathrm{m}}$ ).

Dynamo action occurs primarily where the field is weak, no matter whether in the linear or saturated regime, or whether the flow is laminar or becomes turbulent. The extremely fast growth of this dynamo is mainly due to the stretching ability of the flow. The formation of strong vortex tubes also plays an important role. Stretched magnetic loops are advected by the flow in the vicinity of strong vorticity structures where they become highly twisted and are folded with neighboring field lines of similar polarity. This process increases the magnetic energy exponentially
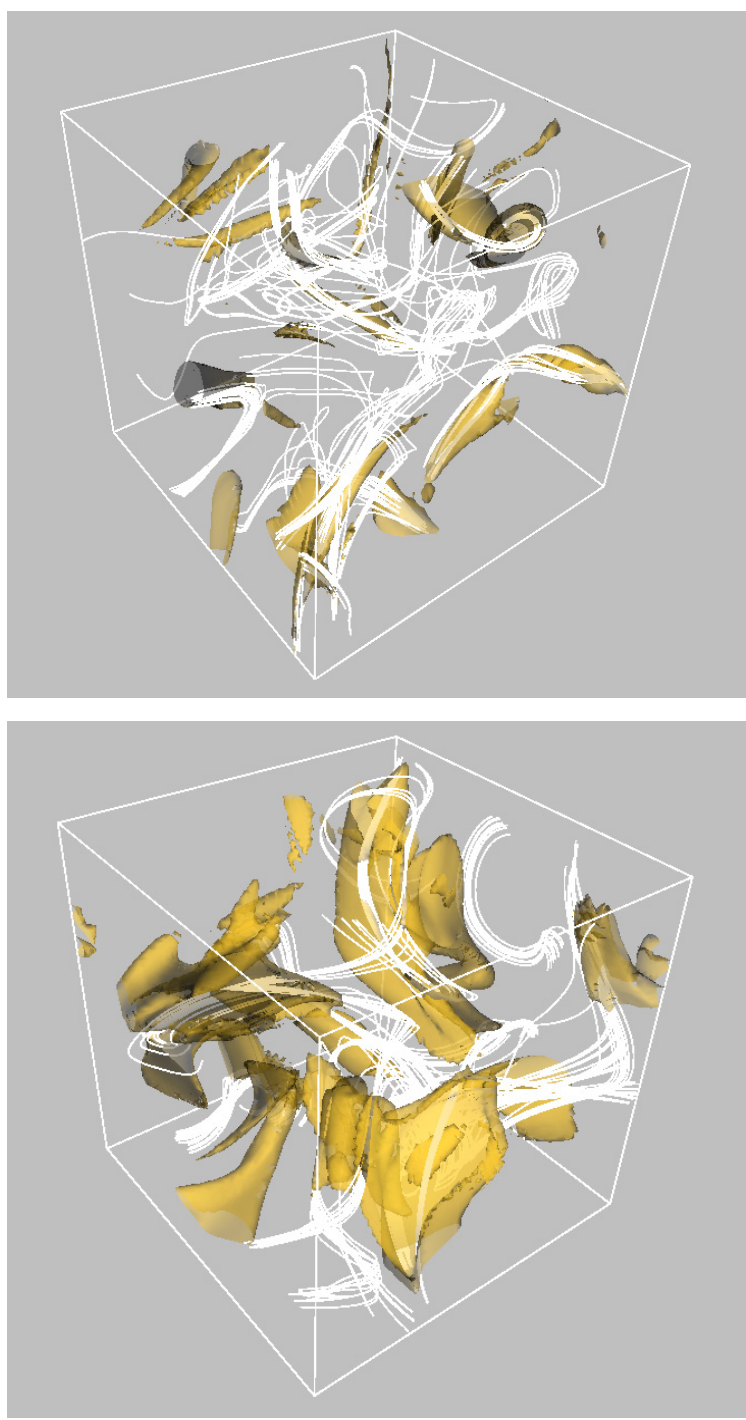

Fig. 14. Top: isosurfaces of the magnetic field and magnetic fieldlines at $t=3861$ for the experiment with $\mathrm{Rm}=400, \mathrm{Re}=100$. Bottom: same visualization for a later time, $t=3888$.

in time. Saturation takes place when the weak field is getting strong enough to force diffusion through the plasma and stops further amplification.

A remarkable result is that this dynamo equilibrates first with approximately equal (scaled) magnetic and velocity fields, which are very similar to the initial flow topology. It does so even for high Reynolds numbers. This solution is an equipartition solution and, as opposed to generic turbulent dynamos in astrophysics, this dynamo has laminar flows and a correspondingly smooth magnetic topology. We have thus illustrated that turbulence is not a necessary condition for a dynamo to generate strong magnetic fields, such as those that are observed in many astrophysical contexts.

We have also demonstrated that after many diffusive time scales the laminar equipartition solution looses its time independent nature. First, the temporal evolution of the energies shows clear oscillations. It seems that the period and amplitude of these oscillations is independent of the magnetic or viscous Reynold numbers. On the other hand, it is likely that these oscillations indicate the operation of another dynamo mode, which is different from the time-independent equipartition mode. Also, it is worthwhile to mention that we find indications of breaking of 
the strong symmetries of the flow during this phase. It is interesting that the disruption of the three-fold symmetries is more effective in experiments with high Reynold numbers and, thus, the system deviates from its initial configuration even before the appearance of the fully developed turbulent phase.

After a period of oscillatory behaviour the system enters a turbulent regime. However, the turbulent mode is not the final mode at saturation, because the system finds again the way to adopt states with high energy levels where kinetic and magnetic energies become almost equal. We find that the saturation of this dynamo suffers from transitions between these modes and, thus, exhibits an intricate dynamical behaviour characterized by laminar and turbulent phases.

The simple initial conditions in our experiments notwithstanding, we conjecture that the building blocks and individual processes involved in the dynamo action and equipartition of the aforementioned dynamo might be of general importance in the studies of astrophysical dynamos. It is an observed fact for example that the Sun possesses a structured, large-scale mean magnetic field of strength comparable to equipartition, which goes through a periodic dynamo process. Thus, studying equipartition dynamos such as the one in the present simulations may be an important step towards the understanding of the fast growth and the subsequent equilibration of astrophysical magnetic fields.

Acknowledgements. Access to computational resources granted by the Danish Center for Scientific Computing is gratefully acknowledged.

\section{References}

Archontis, V. 2000, Ph.D. Thesis, Copenhagen University

Archontis, V., Dorch, S. B. F., \& Nordlund, Å. 2003a, A\&A, 397, 393 Archontis, V., Dorch, S. B. F., \& Nordlund, Å. 2003b, A\&A, 410, 759 Brandenburg, A., \& Subramanian, K. 2005, Phys. Rep., 417, 1

Brandenburg, A., Klapper, I., \& Kurths, J. 1995, Phys. Rev. Lett., 52, 4602

Cameron, R., \& Galloway, D. 2006a, MNRAS, 365, 3

Cameron, R., \& Galloway, D. 2006b, MNRAS, 367, 3

Cattaneo, F., Hughes, D. W., \& Kim, E. 1996, Phys. Rev. Lett., 76, 2057

Childress, S., \& Gilbert, A. D. 1995, Stretch, Twist, Fold: The Fast Dynamo (Berlin: Springer-Verlag), 52

Cowling, T. J. 1934, MNRAS, 140, 39

Dorch, S. B. F. 2000, Phys. Scr., 61, 717

Dorch, S. B. F., \& Archontis, V. 2004, Sol. Phys., 224, 171

Galloway, D., \& O’Brian, N. R. 1993, in Planetary Dynamos (Cambridge), 105 Galloway, D., \& Proctor, M. 1992, Nature, 356, 691

Hyman, J. M. 1972, in Adv. in Comp. Meth. for PDE's-III, ed. R. Vichnevetsky, \& R. S. Stepleman (Publ. IMACS), 313

Mininni, P., Alexakis, A., \& Pouquet, A. 2005, Phys. Rev. E, 72, 4

Mininni, P., Ponty, Y., Montgomery, D. C., et al. 2005, ApJ, 626, 853

Moffatt, H. 1978, Magnetic Field Generation in Electrically Conducting Fluids (Cambridge University Press)

Nordlund, Å., Brandenburg, A., Jennings, R. L., et al. 1992, ApJ, 392, 647

Parker, E. N. 1979, Cosmical Magnetic Fields (Oxford: Clarendon)

Podvigina, O., \& Pouquet, A. 1994, Physica D, 75, 475

Schekochihin, A., Cowley, S., Maron, J., \& Malyshkin, L. 2002, Phys. Rev. E, 65,1

Schekochihin, A., Cowley, S., Taylor, S., Maron, J. \& McWilliams, J. 2004, ApJ, 612,276

Tanner, S., \& Hughes, D. 2003, ApJ, 586, 585

Zienicke, E., Politano, H., \& Pouquet, A. 1998, Phys. Rev. Lett., 81, 4640 